\title{
Transesophageal Pacing Cardiac Induces Cardiac Arrest and Subsequent Brain Injury in Rats
}

\author{
Yingtao Lian ${ }^{1}$, Lan $\mathrm{Yao}^{1}$, Song $\mathrm{Xu}^{1}$, Liping Lu${ }^{1}$, Teng Wang ${ }^{2}$, Guo Hou${ }^{1}$, Yinping $\mathrm{Li}^{3}$, Zhui $\mathrm{Yu}^{1, *}$ \\ ${ }^{1}$ Department of Critical Care Medicine, Renmin Hospital of Wuhan University: Wuhan University Renmin Hospital, Wuhan, China \\ ${ }^{2}$ Department of Cardiovascular, Renmin Hospital of Wuhan University: Wuhan University Renmin Hospital, Wuhan, China \\ ${ }^{3}$ Department of Pathophysiology, Wuhan University School of Basic Medical Sciences, Wuhan, China
}

\section{ARTICLE INFO}

\section{Article History}

Received 25 August 2021

Accepted 17 November 2021

\section{Keywords}

Cardiac pacing

cardiac arrest

cardiopulmonary resuscitation

brain injury

\begin{abstract}
Background: Cardiac Arrest (CA) is one of the leading causes of mortality worldwide. The present study aimed to establish a simple and stable rat model of CA induced by transesophageal cardiac pacing for the investigation of cerebral resuscitation.

Materials and Methods: A total of 26 healthy adult male Sprague-Dawley rats were randomly allocated into two groups: Shamoperated $(n=6)$ and experimental $(n=20)$ groups. High-frequency cardiac pacing $(50 \mathrm{~Hz}, 2 \mathrm{~ms}$ and $30 \mathrm{~V})$ was maintained for $3 \mathrm{~min}$ to induce CA. Providing CA was not achieved, an additional $2 \mathrm{~min}$ of pacing was performed $30 \mathrm{~min}$ later. After $4 \mathrm{~min}$ following the onset of CA, Cardiopulmonary Resuscitation (CPR) was initiated.

Results: CA was successfully induced in all 20 rats by this setting of high-frequency cardiac pacing. Among them, CA was induced in six rats after $2 \mathrm{~min}$ of pacing; the remaining 14 rats underwent CA after $3 \mathrm{~min}$ of pacing. When electrical stimulation was terminated the rate of Pulseless Electrical Activity (PEA) was 85\% (17/20), the rate of Ventricular Fibrillation (VF) was $15 \%(3 / 20)$ and no asystole occurred. Of the 17 PEA rats, 16 were successfully resuscitated and the average duration of CPR was $106.75 \pm 30.81 \mathrm{~s}$. A total of three rats succumbed within $24 \mathrm{~h}$, and one rat succumbed between 24 and $48 \mathrm{~h}$ following successful resuscitation; 12 rats survived to $>72 \mathrm{~h}$. In addition, three rats with VF were successfully resuscitated and the average duration of CPR was $264.33 \pm 130.40 \mathrm{~s}$; one rat succumbed between 24 and $48 \mathrm{~h}$ following successful resuscitation, and two rats survived to $>72 \mathrm{~h}$. The $72 \mathrm{~h}$-survival rate was $74 \%$. No notable injury the esophagus was observed in the rats. Compared with the Sham group, the neurological function of the CA group was significantly impaired $(p<0.05)$; cells in the hippocampal CA1 region of the CA group were significantly damaged $(p<0.05)$.
\end{abstract}

Conclusion: The model of CA induced by transesophageal cardiac pacing in the present study is easy and replicable. Therefore, this model may be used for experimental research into cerebral resuscitation.

(c) 2021 First Affiliated Hospital of Zhengzhou University. Publishing services by Atlantis Press International B.V. This is an open access article distributed under the CC BY-NC 4.0 license (http://creativecommons.org/licenses/by-nc/4.0/).

\section{BACKGROUND}

It was reported that $>356,000$ individuals in the United States [1] and 544,000 individuals in China [2] suffer from Out-of-Hospital Cardiac Arrest (OHCA) annually. In the United States only about $11 \%$ patients survive to discharge; however, poor neurological outcome is often observed. Additionally, only 1-3\% patients who suffer from OHCA in China could survive to discharge. At present, there is no effective treatment for cerebral resuscitation after Cardiac Arrest (CA) other than Target Temperature Management (TTM) which has been reported to have therapeutic effects on cerebral injury following CA in clinical trials [3]. TTM during cerebral resuscitation post-CA has been investigated [3-6]; however, opposing findings have been reported [7-9].

In the past few decades, various models of CA have been employed, including lethal arrhythmia-induced CA types, such as the

“Corresponding author.Email: yuzhui@whu.edu.cn

Peer review under responsibility of the First Affiliated Hospital of Zhengzhou University Data availability statement: The data that support the findings of this study are available from the corresponding author [ZY], upon reasonable request.
Ventricular Fibrillation-induced CA (VFCA) model (54\%), and the asphyxial CA (25\%), potassium chloride-induced CA (8\%) and exsanguination CA models [10]. As various models of CA are used, there is a high degree of heterogeneity; thus, opposing results may be obtained. It is important to establish a stable and reproducible animal model for investigations into organ injury and protection post-CA. Arrhythmia, particularly VF, is the most common cause of sudden patient mortality in clinical situations [11,12]. Therefore, the lethal arrhythmia-induced CA model may be more appropriate for investigations of CA and post-CA syndrome than other models in translational medicine.

It is well reported that rat models of CA are advantageous as they are low cost and easy to apply for screening analysis that requires large quantities of animals. Rat models have been widely used in behavioral studies to assess neurological outcome. Thus, rat models of CA induced by electrical stimulation may be more suitable for studying cerebral resuscitation post-CA. Models of induced-CA have been generated via different approaches, including stimulation of the right ventricular endocardium, or esophagus and chest, and transcutaneous electrical stimulation of the epicardium; however, the experimental procedures are difficult to perform [13-16]. 
Therefore, to investigate brain injury and cerebral resuscitation based on previous reports $[17,18]$, the present study aimed to modify a rat model of CA via transesophageal cardiac pacing.

\section{MATERIALS AND METHODS}

\subsection{Animal Preparation}

The present study was approved by the Institutional Animal Care and Use Committee of Wuhan University (Wuhan, China). All animal experiments were performed according to the National Institutes of Health Guide for the Care and Use of Laboratory Animals. A total of 26 healthy adult male Sprague-Dawley rats (body weight, $300-350 \mathrm{~g})$ were divided into sham-operated $(n=6)$ and experimental groups $(n=20)$; rats were obtained from the Laboratory Animal Unit of Wuhan University. All rats were provided standard chow and housed in a specific pathogen-free room at a constant temperature $\left(20-22^{\circ} \mathrm{C}\right)$ under a 12 -h light/dark cycle. Prior to the experiments, rats were fasted overnight with free access to water.

\subsection{Establishment of the CA Model}

\subsubsection{Anesthesia}

The rats were anesthetized via an intraperitoneal injection of $45 \mathrm{mg} / \mathrm{kg}$ pentobarbital sodium (Figure 1).

\subsubsection{Tracheal intubation}

Tracheal intubation was performed with direct vision using a laryngoscope (Welch-Allyn Pocketscope ${ }^{\text {Tw }}$ Otoscope-Model 22821, Welch Allyn, Inc., NY, USA). Each rat was fixed on a surgical board in supine position with the head bent backwards and the neck extended. Subsequently, the mouth was opened and the tongue was carefully moved with tweezers. The laryngoscope was inserted and adjusted to the correct position. When the glottis was fully visible, a 14-gauge endotracheal tube was inserted into the trachea and the correct position was obtained. Finally, the tube was fixed with a ligature, which was anchored to the surgical board.

\subsubsection{Cannulation via femoral arterial puncture and venipuncture}

The fur of left groin of rats was shaved and the area was prepared for surgery using iodophor at least three times. An incision was made and the femoral artery was dissociated. A catheter (polyethylene catheter, PE50) filled with $0.9 \%$ saline solution containing $5 \mathrm{IU} / \mathrm{ml}$ of heparin was inserted into left femoral artery and connected with a stopcock, which were respectively connected to an injector with heparinized $0.9 \%$ saline solution and a highsensitivity pressure transducer. A 24-gauge close-vein indwelling needle containing $0.9 \%$ saline solution was inserted into the left femoral vein for drug administration. After cannulating the left femoral artery and vein, at least one Arterial Blood Gas (ABG) analysis for $\mathrm{pH}, \mathrm{pO}_{2}, \mathrm{pCO}_{2}, \mathrm{HCO}_{3}^{-}$, Base Excess (BE) and lactate (epoc Blood Analysis, Siemens, Ottawa, Canada) was performed in order to achieve steady-state conditions.

\subsubsection{Insertion of the transesophageal stimulating electrode}

A 5-F coronary sinus electrode catheter [Johnson \& Johnson medical (Shanghai) Ltd., Shanghai, China] and two 2-mm ring electrodes, with an inter-electrode distance of $5 \mathrm{~mm}$, was orally inserted into the esophagus of the rats. The tip of the catheter was placed to a depth about $7 \mathrm{~cm}$ from the incisors and positioned to ensure constant ventricular capture; the position of the catheter was adjusted according to rat size. Conventional lead II Electrocardiograms (ECG) were recorded via subcutaneous needle electrodes. The core temperature was monitored continuously using a rectal probe and maintained at $37^{\circ} \mathrm{C}$ with a heat lamp.

The ECG and femoral artery pressure were consistently monitored and recorded with LabChart7.0 [PowerLab 16/30ML880, AD Instruments international trade (Shanghai) Co., Ltd., Shanghai, China].

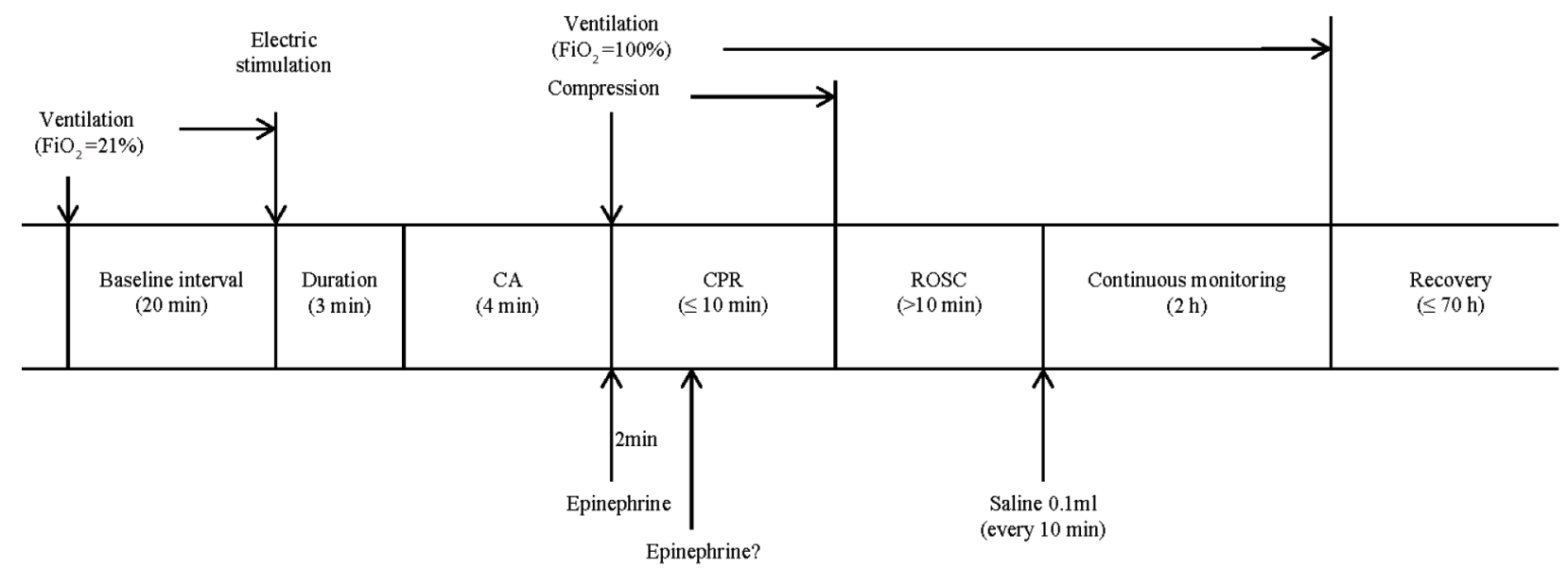

Figure 1 Experimental timeline. Arrows indicate the time points of experimental interventions. $\mathrm{FiO}_{2}$, fraction of inspired oxygen. 


\subsubsection{Induction of $\mathrm{CA}$}

Rats were mechanically ventilated at a rate of 70 breaths per minute via a volume-controlled mode using a small animal ventilator (VentStarR415, RWD Life Science Co., Ltd., Shenzhen, China). The tidal volume was $7 \mathrm{ml} / \mathrm{kg}$. Mechanical Ventilation (MV) continued for $\geq 20$ min to ensure the stable conditions of rats were maintained, but was discontinued prior to CA induction. CA was induced by transesophageal cardiac pacing with high-frequency stimulation $(50 \mathrm{~Hz}$; pulse duration, $2 \mathrm{~ms}$ and intensity, $30 \mathrm{~V}$ ) using a stimulator (S88; Grass Instruments, Quincy, MA, USA) for about 3 min (Figure 2A). When cardiac pacing was conducted, the electrode was adjusted to ensure the tip of the electrode was close to the heart. CA was defined as follows [19]: (1) The arterial pulse wave was not observed and the Mean Arterial Pressure (MAP) was <25 mmHg; and (2) the ECG wave changed from normal to that of Pulseless Electrical Activity (PEA) (Figure 2B), VF (Figure 2C) or asystole following the termination of electrical stimulation. Generally, CA can be induced after 3 min of electrical stimulation in rats. Providing CA was not achieved, an additional $2 \mathrm{~min}$ of pacing was performed

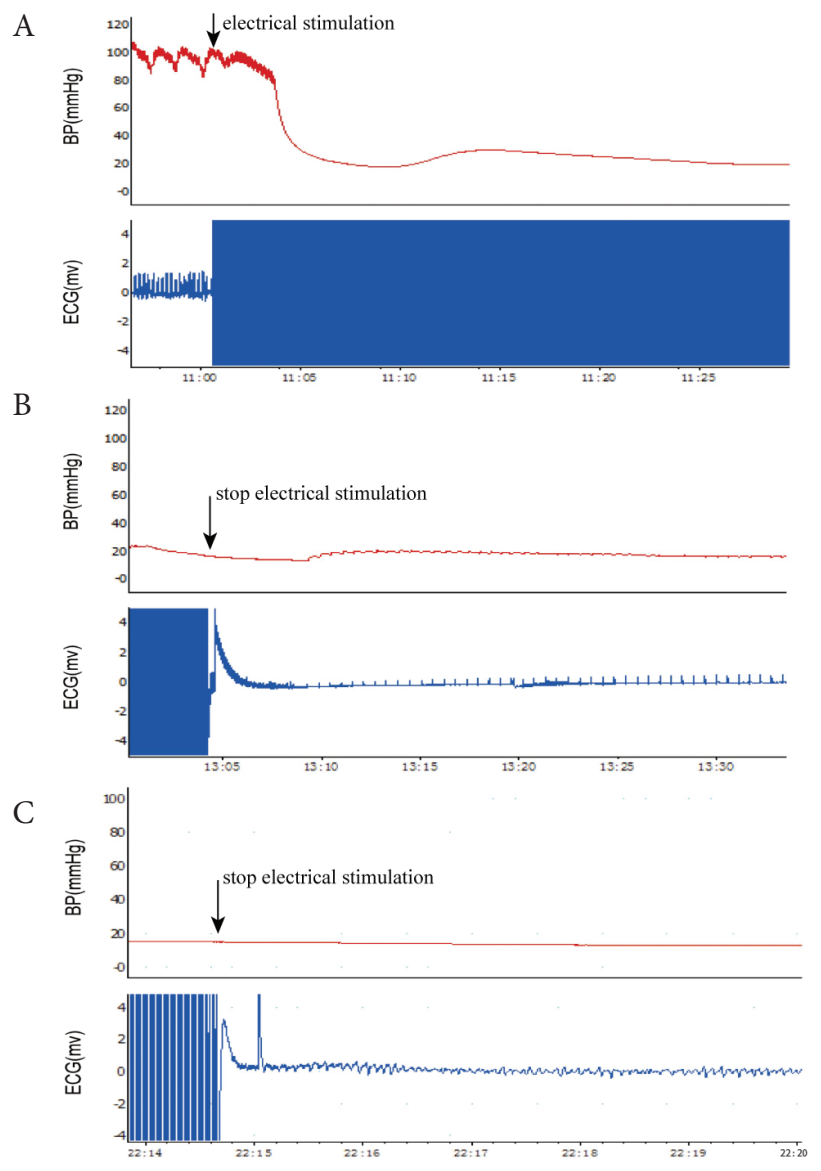

Figure 2 ECG and BP changes in CA. (A) High-frequency pacing induced a decrease in BP and loss of pulse; (B) The ECG wave changed to that of pulseless electrical activity and the mean arterial pressure was $<25 \mathrm{mmHg}$ following the termination of electrical stimulation.

(C) The ECG wave changed to that of ventricular fibrillation and the mean arterial pressure was $<25 \mathrm{~mm} \mathrm{Hg}$ following the termination of electrical stimulation.
30 min later. The initiation of CA was determined from when the MAP $<25 \mathrm{mmHg}$ and the arterial pulse was undetected. The rats were observed without intervention for $4 \mathrm{~min}$. The induction of CA was considered to fail providing the autonomous cardiac rhythm returned and the MAP $>25 \mathrm{mmHg}$ during the observation period.

\subsection{Cardiopulmonary Resuscitation and Treatments following the Return of Spontaneous Circulation}

Cardiopulmonary Resuscitation (CPR) was initiated 4 min post-CA. Ventilation was conducted using a ventilator with the following parameters: The respiratory frequency was 70 times/ min, the tidal volume was $7 \mathrm{ml} / \mathrm{kg}$ and the fraction of inspired oxygen was $100 \%$. External chest compressions were synchronized with ventilation and always performed by the same investigator using a metronome; a rate of 200 compressions per minute with an equal duration of compression-relaxation was applied. The compression depth was one-third of the anteroposterior chest diameter. A dose of adrenalin $(200 \mu \mathrm{g} / \mathrm{kg})$ was administered to the rats at the beginning of $\mathrm{CPR}$ and repeated 2 min later as required. Return of Spontaneous Circulation (ROSC) can be defined as the restoration of the supraventricular cardiac rhythm with a MAP of $\geq 60 \mathrm{mmHg}$ for a minimum of $10 \mathrm{~min}$. Providing ROSC was not achieved after 10 min with the aforementioned strategy, CPR was regarded as unsuccessful and the efforts of resuscitation were terminated. The $\mathrm{ABG}$ analysis for $\mathrm{pH}, \mathrm{pO}_{2}, \mathrm{pCO}_{2}, \mathrm{HCO}_{3}{ }^{-}, \mathrm{BE}$ and lactate were tested and recorded again at $10 \mathrm{~min}$ after ROSC. Rats that were successfully resuscitated continued to be monitored for $2 \mathrm{~h}$ with an ECG; the femoral artery pressure was also recorded. $\mathrm{MV}$ was continued as the autonomous respiration of the rats was weak. Saline solution $(0.1 \mathrm{ml}, 0.9 \%)$ was infused every $10 \mathrm{~min}$ and administration during this period was limited to $2 \mathrm{ml}$. After $2 \mathrm{~h}$ of observation, MV was withdrawn, all tubes were removed and wounds were sutured. Each rat was fed in a separate cage with free access to food and water. The duration of survival following ROSC was recorded up to $72 \mathrm{~h}$. Rats that had survived following analysis were sacrificed and examined for potential damage resulting from the operations.

The rats were randomized into two groups: Sham $(n=6)$ and CA ( $n=20)$ groups. Rats of the Sham group underwent all the same operations as aforementioned without the induction of CA.

\subsection{Neurological Functional Examination}

Neurological Deficit Scores (NDS) were assessed at 24 and $72 \mathrm{~h}$ following CPR. The NDS were measured by two investigators as previously described [20]; the investigators were blinded to the experimental groups. Briefly, the total NDS comprised the following components: General behavioral function (consciousness and respiration, 40 points), motor function (10 points), cranial nerve function (20 points), coordination (20 points), and sensory function (10 points). The scoring system ranged from 0 (normal brain function) to 100 (brain death). In this study, dead rats were not included in the NDS assessment. 


\subsection{HE Staining}

The rats were anesthetized and intracardially perfused with normal saline, followed by $4 \%$ paraformaldehyde. After decapitation, the brains were removed and the tissues at the coronal plane from 1 to $4 \mathrm{~mm}$ posterior to the optic chiasma were harvested. For Hematoxylin-Eosin (HE) staining, coronal sections with $4 \mathrm{~mm}$ thickness were cut by microtome (Leica CM3050S, Solms, Germany), then stained with hematoxylin-eosin. The sections of stratum pyramidale of hippocampal CA1 were observed using a light microscope (Olympus, Tokyo, Japan). Ten fields of vision (magnification of $40 \times$ ) in the hippocampal CA1 area were randomly sampled from each brain tissue sample, and the HE stained neuronal cells were counted under an optical microscope.

\subsection{Statistical Analysis}

All data were expressed as the mean \pm standard deviation and the statistical analyses were performed with SPSS version 20.0 (IBM Corp., Armonk, NY, USA). An unpaired $t$-test was used to compare the same parameters between two groups. Mann-Whitney $U$-test was employed to compare the neurological deficit scores between groups. The survival rates between two groups were obtained using Kaplan-Meier survival analysis and compared with a log-rank test. $p<0.05$ was considered to indicate a statistically significant difference.

\section{RESULTS}

\subsection{Baseline Characteristics of Animals}

In the pre-cardiac arrest period (baseline) following animal preparation, no significant differences were observed between the groups regarding body weight, hemodynamics and ABG variables (Table 1).

\subsection{Outcomes of Induction of CA and CPR}

In the present study, CA was successfully induced in all 20 rats by high-frequency cardiac pacing. Among them, CA was induced in six rats following pacing for $2 \mathrm{~min}$; the other 14 rats exhibited CA

Table 1 Baseline characteristics

\begin{tabular}{lccc}
\hline Variables & Sham & CA & $\boldsymbol{p}$ \\
\hline$N$ & 6 & 20 & - \\
Body weight $(\mathrm{g})$ & $336.17 \pm 5.31$ & $338.40 \pm 9.45$ & 0.588 \\
Heart rate $(\mathrm{beat} / \mathrm{min})$ & $440.33 \pm 27.61$ & $446.60 \pm 19.32$ & 0.621 \\
$\mathrm{MAP}(\mathrm{mmHg})$ & $125.33 \pm 11.36$ & $127.95 \pm 11.38$ & 0.626 \\
$\mathrm{pH}$ & $7.40 \pm 0.38$ & $7.40 \pm 0.26$ & 0.931 \\
$\mathrm{pO}_{2}(\mathrm{mmHg})$ & $126.00 \pm 3.90$ & $125.35 \pm 5.23$ & 0.782 \\
$\mathrm{pCO}_{2}(\mathrm{mmHg})$ & $35.83 \pm 2.31$ & $34.45 \pm 7.58$ & 0.667 \\
$\mathrm{HCO}_{3}^{-}(\mathrm{mmol} / \mathrm{L})$ & $23.75 \pm 0.92$ & $24.18 \pm 1.22$ & 0.439 \\
$\mathrm{BE}(\mathrm{mmol} / \mathrm{L})_{\mathrm{Lactate}(\mathrm{mmol} / \mathrm{L})}$ & $0.38 \pm 1.09$ & $-0.21 \pm 1.53$ & 0.387 \\
\hline
\end{tabular}

Values are mean $\pm \mathrm{SD}$. MAP, mean arterial pressure; $\mathrm{pO}_{2}$, partial pressure of oxygen; $\mathrm{pCO}_{2}$, partial pressure of carbon dioxide; $\mathrm{HCO}_{3}{ }^{-}$, bicarbonate radical; $\mathrm{BE}$, base excess. after 3 min of pacing. When electrical stimulation was terminated, the rate of PEA was $85 \%(17 / 20)$, the rate of VF was $15 \%(3 / 20)$ and no asystole occurred. No automatic cardioversion phenomenon were observed during the non-intervention period. Of the 17 PEA rats, 16 were successfully resuscitated and the average duration of CPR was $106.75 \pm 30.81 \mathrm{~s}$. A total of three rats succumbed within $24 \mathrm{~h}$, and one rat succumbed between 24 and $48 \mathrm{~h}$ following successful resuscitation; the other 12 rats survived to $>72 \mathrm{~h}$. In addition, three rats with VF were successfully resuscitated and the average duration of CPR was $264.33 \pm 130.40 \mathrm{~s}$; one rat succumbed between 24 and $48 \mathrm{~h}$ following successful resuscitation, and two rats survived to $>72 \mathrm{~h}$. The frequency of intravenous injection of adrenaline was $1.53 \pm 0.84$ times. The $72 \mathrm{~h}$-survival rate of the CA group was $74 \%(14 / 19)$ and that of the Sham group was $100 \%$ $(6 / 6)$. The $72 \mathrm{~h}$-survival rate of the CA group was not significantly lower compared with the Sham group $(p=0.183)$ (Figure 3 and Table 2). No notable damage to the heart or lungs was observed during autopsy; no thermal injury of esophagus was detected in any of the rats.

\subsection{Hemodynamic and ABG Studies}

The heart rate, $\mathrm{MAP}, \mathrm{pH}, \mathrm{HCO}_{3}{ }^{-}$and $\mathrm{BE}$ were significantly decreased whereas $\mathrm{pO}_{2}, \mathrm{pCO}_{2}$ and lactate were significantly increased following the resuscitation of rats in the CA group compared with the Sham group (Figure 4 and Table 3).

\subsection{Neurological Deficit Scores}

The NDS of the Sham group was 0 . The NDS of the CA group at 24 and $72 \mathrm{~h}$ post-CA were significantly increased compared with the Sham group ( $p<0.05$; Figure 5).

\subsection{Histological Examination of the Hippocampus by HE Staining}

In the hippocampal CA1 region of the Sham group, cell morphology was notably visible and cell structure was compact with abundant cytoplasm. In the CA group, cells were sparsely arranged and

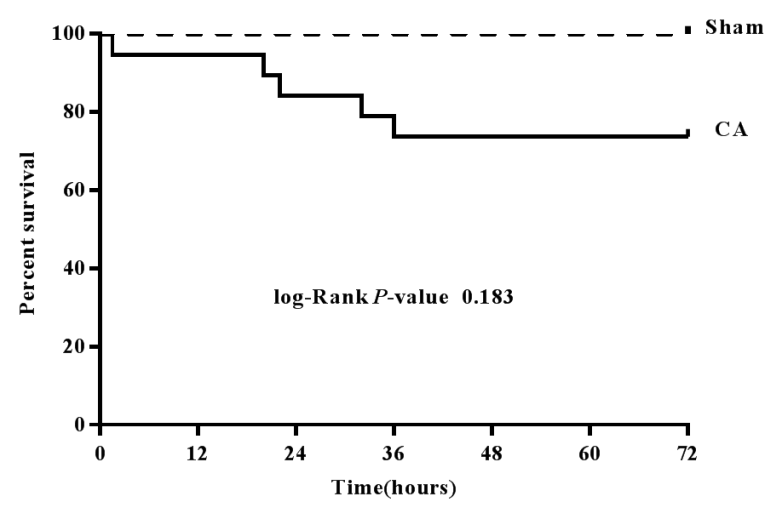

Figure 3 Survival rate during $72 \mathrm{~h}$ of recovery following the return of spontaneous circulation. Dashed line, Sham group, $n=6$; Solid line, cardiac arrest group, $n=19$. 
Table 2 The data for the model of cardiac arrest in rats

\begin{tabular}{rccccccc}
\hline SN & ICAT (min) & CREES & CRBCPR & DOCPR (s) & AF & ST (h) & Complication \\
\hline 1 & 3 & PEA & PEA & 126 & 1 & 1.5 & None \\
2 & 3 & PEA & AS & 190 & 2 & 22 & None \\
3 & 3 & PEA & PEA & 85 & 1 & $>72$ & None \\
4 & 2 & PEA & PEA & 95 & 1 & $>72$ & None \\
5 & 3 & PEA & PEA & 100 & 1 & $>72$ & None \\
6 & 3 & PEA & AS & 104 & 2 & $>72$ & None \\
7 & 2 & PEA & PEA & 109 & 1 & $>72$ & None \\
8 & 3 & PEA & AS & 84 & 1 & 36 & None \\
9 & 2 & PEA & PEA & 85 & 1 & $>72$ & None \\
10 & 3 & PEA & AS & 111 & 2 & $>72$ & None \\
11 & 2 & PEA & PEA & 75 & 1 & $>72$ & None \\
12 & 3 & PEA & AS & 148 & 2 & $>72$ & None \\
13 & 3 & PEA & AS & 99 & 1 & $>72$ & None \\
14 & 3 & PEA & PEA & 76 & 1 & 20 & None \\
15 & 2 & VF & PEA & 282 & 3 & $>72$ & None \\
16 & 3 & VF & VF & 385 & 4 & 32 & None \\
17 & 3 & VF & PEA & 126 & 1 & $>72$ & None \\
18 & 3 & PEA & PEA & 138 & 2 & $>72$ & None \\
19 & 2 & PEA & PEA & 83 & 1 & $>72$ & None \\
20 & 3 & PEA & PEA & $>600$ & 5 & 0 & None \\
\hline
\end{tabular}

ICAT, induced CA time; CREES, cardiac rhythm at end of electric stimulation; CRBCPR, cardiac rhythm at the beginning of CPR; DOCPR, duration of CPR; AF, administration frequency; ST, survival time.
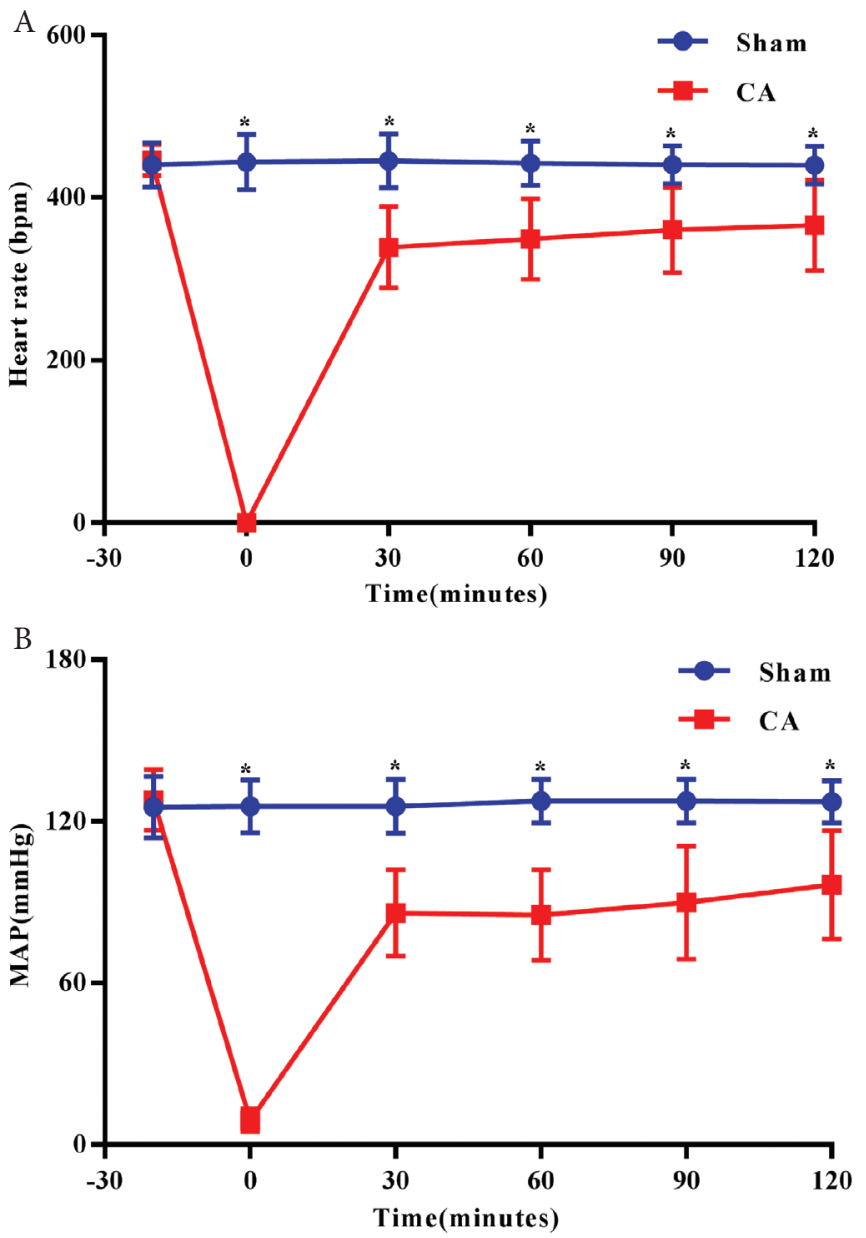

Figure 4 Alterations in heart rate and MAP. (A) Heart rate; (B) MAP. ${ }^{*} p<0.05$ vs. Sham group. Data are presented as the mean \pm standard deviation. -30 indicates $30 \mathrm{~min}$ prior to the induction of CA.
Table 3 Physiological variables at $10 \mathrm{~min}$ after ROSC

\begin{tabular}{lccc}
\hline Variables & Sham & CA & $\boldsymbol{p}$ \\
\hline$N$ & 6 & 19 & - \\
$\mathrm{pH}$ & $7.41 \pm 0.03$ & $7.22 \pm 0.05$ & 0.000 \\
$\mathrm{pO}_{2}(\mathrm{mmHg})$ & $126.67 \pm 2.58$ & $234.89 \pm 46.05$ & 0.000 \\
$\mathrm{pCO}_{2}(\mathrm{mmHg})$ & $36.50 \pm 2.26$ & $42.37 \pm 1.54$ & 0.001 \\
$\mathrm{HCO}_{3}^{-}(\mathrm{mmol} / \mathrm{L})$ & $24.28 \pm 1.19$ & $16.93 \pm 1.65$ & 0.000 \\
$\mathrm{BE}(\mathrm{mmol} / \mathrm{L})$ & $0.42 \pm 1.5$ & $-13.97 \pm 1.40$ & 0.000 \\
Lactate $(\mathrm{mmol} / \mathrm{L})$ & $1.27 \pm 0.21$ & $8.50 \pm 0.90$ & 0.000 \\
\hline \multicolumn{4}{l}{ Values are mean $\pm \mathrm{SD}^{2} \mathrm{pO}_{2}$, partial pressure of oxygen; $\mathrm{pCO}_{2}$, partial pressure of } \\
carbon dioxide; $\mathrm{HCO}_{3}^{-}$, bicarbonate radical; $\mathrm{BE}$, base excess.
\end{tabular}

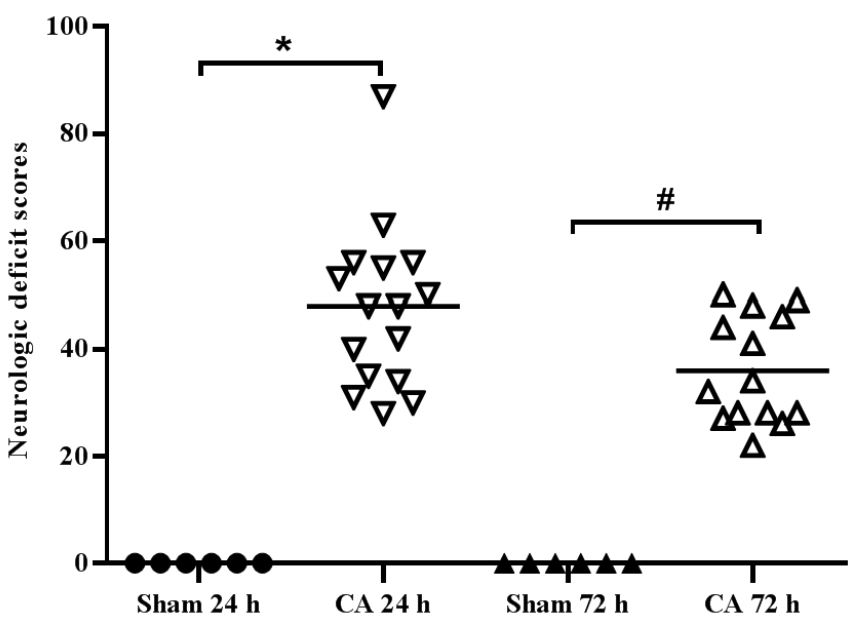

Figure 5 Neurological function significantly decreases following cardiac arrest in rats. Neurological deficits scores were measured at 24 and $72 \mathrm{~h}$ following the return of spontaneous circulation. The NDS ranged from 0 (normal brain function) to 100 (brain death). ${ }^{*} p<0.05$ vs. Sham group, $\# p<0.05$ vs. CA $24 \mathrm{~h}$. 
cell morphology was unclear (Figure 6A). Compared with the Sham group, there were more cells exhibiting eumorphism and the number of vital neurons was significantly reduced $(p<0.05$; Figure 6B).

\section{DISCUSSION}

Previous studies have suggested that OHCA is mainly caused by malignant arrhythmia; VF and pulseless ventricular tachycardia were reported to be the most common cause of primary sudden OHCA $[12,21]$.

Thus, various electrical stimulation approaches have been employed to induce CA. As early as 1988, von Planta et al. [13] developed a rat model of VFCA, which was induced by an alternating current delivered to the right ventricular endocardium; however, only eight of 14 rats were successfully resuscitated after $4 \mathrm{~min}$ of CA and the operative technique is complex. In 1997, Böttiger et al. [14] developed an animal model induced via an esophageal electrode and an external electrode placed on the chest of the rats. This model was the first to induce VFCA by esophageal pacing. The experimental approach is less complex with slight surgical trauma; $50 \%$ rats exhibited ROSC during CA. As the precise location for placing the esophageal electrode and the duration for cardiac pacing remain unclear, further investigation is required to improve the reproducibility of this model. In 2007 , Chen et al. $[17,18]$ successfully established a model of VFCA in rats induced by transesophageal cardiac pacing; however, this procedure was associated with significant surgical trauma. In addition, the resuscitated rats were monitored for $30 \mathrm{~min}$ following ROSC and there was a lack of a Sham group in these studies. Whether this model can be used for investigations into brain injury and cerebral resuscitation post-CA remains unknown. To perform long-term research into brain injury and protection post-CA, a model of CA with reduced procedure-associated trauma should be used. For this purpose, the present study aimed to develop a stable, easy and reproducible animal model of CA.

The present study attempted to reduce unnecessary operative trauma and simplify the operative techniques. An electrode was inserted into the esophagus rather than the right ventricular endocardium or epicardium, as conducted in other studies $[13,16]$. Additionally, intratracheal intubation under direct vision is superior to traditional tracheotomy, as risks of bleeding, infection and injuries to the respiratory tract are lower. Furthermore, it is easier to insert a catheter into the femoral artery or femoral vein than into the carotid artery or external jugular vein. Importantly, insert a catheter into femoral artery or femoral vein does not injure the vasculature in the neck and has no effect on the blood flow supplying the brain. The NDS and histological analysis of the hippocampus indicated that, compared with the Sham group, the neurological function of the CA group was significantly impaired. Therefore, the model employed in the present study may be more suitable for studying brain injury and cerebral resuscitation post-CA. Thus, compared with the existing animal models of CA, our method has the advantages of reduced surgical trauma, simplicity, replicability and fewer complications. As the resting heart rate is very high, the refractory period is correspondingly short [22-24] and it is difficult to maintain continuous VF. Accordingly, 3 min of continuous electrical stimulation was used to prevent the phenomenon of spontaneous defibrillation. After 3 min of high-frequency burst pacing, CA was induced successfully in all 20 rats. Interestingly, the rate of PEA was 17/20 in the present study, which differed to that reported by Chen et al. [18]; however, regardless of PEA or VF, the main effect is a failure of the heart to maintain various types of forward blood flow, including coronary, systemic, and pulmonary blood flow. In addition, with effective intervention, the average duration of CPR in PEA rats was shorter than rats with VF; electrical defibrillation was not required in PEA rats during resuscitation. Of the 20 rats, 19 were successfully resuscitated and the average duration of CPR was $131.63 \pm$ $78.52 \mathrm{~s}$, which was shorter than reported by Chen et al. [18].

In the present study, compared with the Sham group, the heart rate and mean arterial pressure decreased significantly following ROSC in the CA group, but increased spontaneously with progression of the disease. After $2 \mathrm{~h}$ following treatment, near normal levels were recorded. These findings indicated that the rats exhibited cardiovascular dysfunction post-CA, which was similar to previous studies [25-27]. Cardiovascular function decreased following CA, but gradually recovered by active $\mathrm{CPR}$ and treatments post-CA. The recovery process of CPR promotes blood perfusion of the vital organs, which supports the effectiveness of rescues and treatments administered post-CA in the present study. The changes of ABG variables at $10 \mathrm{~min}$ after ROSC of rats in the CA group are consistent with clinical phenomenon and indicated the success of our CA model.
A

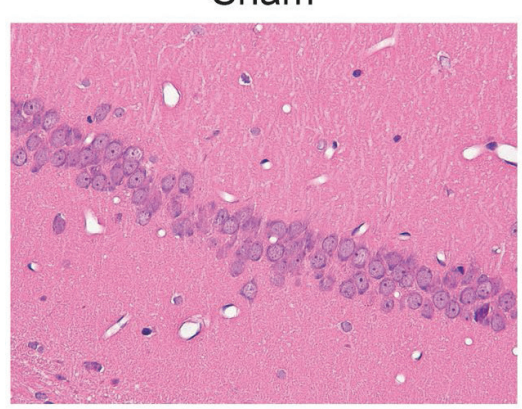

Sham

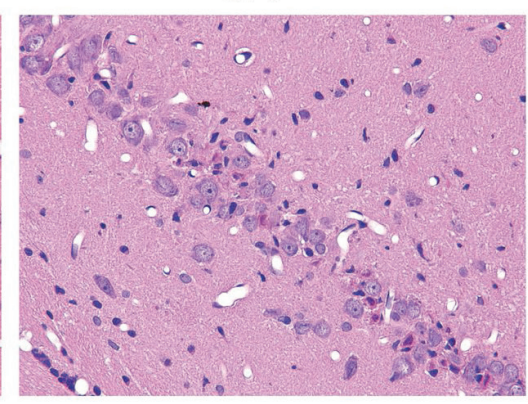

B

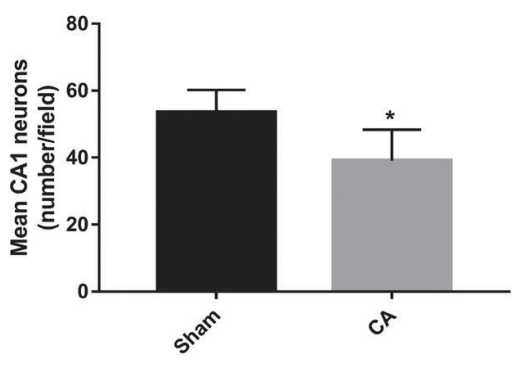

Figure 6 Hippocampal CA1 neurons at $72 \mathrm{~h}$ following the return of spontaneous circulation. (A) Representative images of HE staining of the hippocampal CA1 neurons $(\times 400)$. Neural cells arrange orderly with complete cell structure in Sham group. Cells were sparsely arranged and cell morphology was unclear in CA group. (B) Quantification of the hippocampal CA1 neurons by HE staining, date are shown as mean \pm SD, ${ }^{*} p<0.05$ vs. Sham group. 
There are some limitations of the present study. First, it was a small animal study performed on healthy rats, whereas most patients with CA have concurrent underlying disease $[9,28]$. Therefore, it cannot be extrapolated to large animal or human and other etiology of CA. Second, we only evaluated the survival situation at $72 \mathrm{~h}$, so our model may not be applicable to long-term survival study.

\section{CONCLUSION}

In summary, the present study was conducted in a preliminary manner; however, there are certain limitations. The rat model of CA induced by transesophageal cardiac pacing is advantageous as of slight surgical trauma, and is a simple procedure that is easy to duplicate with few complications and long-term survival. In particular, vasculature in the neck of animal models are intact throughout the procedure and blood flow to the brain is not affected by surgery. Therefore, this modified rat model of CA may be suitable for long-term investigations into brain injury and cerebral resuscitation post-CA, and may be used in the translational medicine for the treatment of CA.

\section{CONFLICTS OF INTEREST}

The authors declare they have no conflicts of interest.

\section{AUTHORS' CONTRIBUTION}

All authors have made significant contribution to the studies. Zhui Yu, Yinping Li designed the studies and reviewed of the manuscript. Yingtao Lian, Lan Yao, Song Xu, Liping Lu and Teng Wang conducted the experiments and analyzed the data. Yingtao Lian, Zhui Yu wrote the manuscript.

\section{ACKNOWLEDGEMENTS}

The present study was supported by grants from the National Natural Science Foundation of China [grant no. 81772039]. We wish to thank the efforts of all the members involved in the present study for their help in conducting the experiments and data interpretation. This study was performed at the Cardiovascular Research Institute of Wuhan University (Wuhan, China), we also thank all those who helped us in the lab.

\section{REFERENCES}

[1] Benjamin EJ, Blaha MJ, Chiuve SE, Cushman M, Das SR, Deo R, et al. Heart disease and stroke statistics_-2017 update: a report from the American heart association. Circulation 2017; 135;e146-e603.

[2] Zhang S. Sudden cardiac death in China: current status and future perspectives. EP Europace 2015;17;ii14-ii18.

[3] The Hypothermia after Cardiac Arrest Study Group. Mild therapeutic hypothermia to improve the neurologic outcome after cardiac arrest. N Engl J Med 2002;346;549-56.

[4] Arrich J, Holzer M, Havel C, Müllner M, Herkner H. Hypothermia for neuroprotection in adults after cardiopulmonary resuscitation. Cochrane Database Syst Rev 2016;2;CD004128.
[5] Bernard SA, Gray TW, Buist MD, Jones BM, Silvester W, Gutteridge G, et al. Treatment of comatose survivors of out-of-hospital cardiac arrest with induced hypothermia. N Engl J Med 2002;346;557-63.

[6] Nielsen N, Hovdenes J, Nilsson F, Rubertsson S, Stammet P, Sunde $\mathrm{K}$, et al. Outcome, timing and adverse events in therapeutic hypothermia after out-of-hospital cardiac arrest. Acta Anaesthesiol Scand 2009;53;926-34.

[7] Nielsen N, Friberg H, Gluud C, Herlitz J, Wetterslev J. Hypothermia after cardiac arrest should be further evaluated-a systematic review of randomised trials with meta-analysis and trial sequential analysis. Int J Cardiol 2011;151;333-41.

[8] Fisher GC. Hypothermia after cardiac arrest: feasible but is it therapeutic?. Anaesthesia 2008;63;885-6; author reply 886.

[9] Nielsen N, Wetterslev J, Cronberg T, Erlinge D, Gasche Y, Hassager $\mathrm{C}$, et al. Targeted temperature management at $33^{\circ} \mathrm{C}$ versus $36^{\circ} \mathrm{C}$ after cardiac arrest. N Engl J Med 2013;369;2197-206.

[10] Vognsen M, Fabian-Jessing BK, Secher N, Løfgren B, Dezfulian C, Andersen LW, et al. Contemporary animal models of cardiac arrest: a systematic review. Resuscitation 2017;113;115-23.

[11] Tang PT, Shenasa M, Boyle NG. Ventricular arrhythmias and sudden cardiac death. Card Electrophysiol Clin 2017;9;693-708.

[12] Engdahl J, Holmberg M, Karlson BW, Luepker R, Herlitz J. The epidemiology of out-of-hospital 'sudden' cardiac arrest. Resuscitation 2002;52;235-45.

[13] von Planta I, Weil MH, von Planta M, Bisera J, Bruno S, Gazmuri RJ, et al. Cardiopulmonary resuscitation in the rat. J Appl Physiol (1985) $1988 ; 65 ; 2641-7$.

[14] Böttiger BW, Krumnikl JJ, Gass P, Schmitz B, Motsch J, Martin E. The cerebral 'no-reflow' phenomenon after cardiac arrest in ratsinfluence of low-flow reperfusion. Resuscitation 1997;34;79-87.

[15] Kofler J, Hattori K, Sawada M, DeVries AC, Martin LJ, Hurn PD, et al. Histopathological and behavioral characterization of a novel model of cardiac arrest and cardiopulmonary resuscitation in mice. J Neurosci Methods 2004;136;33-44.

[16] Lin Jy, Liao Xx, Li H, Wei Hy, Liu R, Hu Cl, et al. Model of cardiac arrest in rats by transcutaneous electrical epicardium stimulation. Resuscitation 2010;81;1197-204.

[17] Chen MH, Liu TW, Xie L, Song FQ, He T, Zeng Zy, et al. A simpler cardiac arrest model in rats. Am J Emerg Med 2007;25; 623-30.

[18] Chen MH, Liu TW, Xie L, Song FQ, He T, Zeng ZY, et al. Ventricular fibrillation induced by transoesophageal cardiac pacing: a new model of cardiac arrest in rats. Resuscitation $2007 ; 74 ; 546-51$

[19] Idris AH, Becker LB, Ornato JP, Hedges JR, Bircher NG, Chandra $\mathrm{NC}$, et al. Utstein-style guidelines for uniform reporting of laboratory CPR research. A statement for healthcare professionals from a task force of the American Heart Association, the American College of Emergency Physicians, the American College of Cardiology, the European Resuscitation Council, the Heart and Stroke Foundation of Canada, the Institute of Critical Care Medicine, the Safar Center for Resuscitation Research, and the Society for Academic Emergency Medicine. Writing Group. Circulation 1996;94;2324-36.

[20] Katz L, Ebmeyer U, Safar P, Radovsky A, Neumar R. Outcome model of asphyxial cardiac arrest in rats. J Cereb Blood Flow Metab 1995;15;1032-9.

[21] Atwood C, Eisenberg MS, Herlitz J, Rea TD. Incidence of EMStreated out-of-hospital cardiac arrest in Europe. Resuscitation $2005 ; 67 ; 75-80$. 
[22] Papadimitriou D, Xanthos T, Dontas I, Lelovas P, Perrea D. The use of mice and rats as animal models for cardiopulmonary resuscitation research. Lab Anim 2008;42;265-76.

[23] Damiano RJ, Asano T, Smith PK, Cox JL. Effect of the right ventricular isolation procedure on ventricular vulnerability to fibrillation. J Am Coll Cardiol 1990;15;730-6.

[24] Manoach M. Factors influencing maintenance and spontaneous termination of ventricular fibrillation. Int J Cardiol 1984;5; 398-402.

[25] Varvarousis D, Varvarousi G, Iacovidou N, D’Aloja E, Gulati A, Xanthos T. The pathophysiologies of asphyxial vs dysrhythmic cardiac arrest: implications for resuscitation and post-event management. Am J Emerg Med 2015;33;1297-304.
[26] Uray T, Lamade A, Elmer J, Drabek T, Stezoski JP, Missé A, et al. Phenotyping cardiac arrest: bench and bedside characterization of brain and heart injury based on etiology. Crit Care Med 2018;46;e508-e15.

[27] Fumagalli F, Russo I, Staszewsky L, Li Y, Letizia T, Masson S, et al. Ranolazine ameliorates postresuscitation electrical instability and myocardial dysfunction and improves survival with good neurologic recovery in a rat model of cardiac arrest. Heart Rhythm 2014;11;1641-7.

[28] Granfeldt A, Wissenberg M, Hansen SM, Lippert FK, LangJensen T, Hendriksen OM, et al. Clinical predictors of shockable versus non-shockable rhythms in patients with out-of-hospital cardiac arrest. Resuscitation 2016;108;40-7. 